\title{
ORDINI RELIGIOSI E POLITICA REGIA NELLA SARDEGNA CATALANO-ARAGONESE DELLA PRIMA METÀ DEL XIV SECOLO
}

\author{
Maria Giuseppina Meloni \\ Istituto sui Rapporti italo-iberici \\ (CNR, Cagliari, Italia)
}

\section{SOMMARIO}

1. Gli Ordini Mendicanti.- 2. Gli Ordini Monastici Benedettini.

Già durante le trattative diplomatiche che precedettero l'inizio della conquista del Regnum Sardiniae et Corsicae, il sovrano catalano Giacomo II e suo figlio Alfonso si mostrarono perfettamente consapevoli dell'importanza dell'appoggio del clero per il buon esito dell'impresa che si accingevano ad affrontare ${ }^{1}$. Le difficoltà incontrate durante la guerra per l'acquisizione dei territori sardi appartenenti al Comune di Pisa, i problemi subito sorti nei rapporti con le entità politiche presenti nell'isola e il precario

\footnotetext{
'Sulle trattative diplomatiche precedenti la conquista e l'atteggiamento filoaragonese di una parte del clero surdo V. SALAVERT Y ROCA, Cerdeña y la expansion mediterranea de la Coroma de Aragon, 2 voll., Madrid 1956, in particolare il vol. I, pp. 311 e ss.; $M$. TANGHERONI, Alcuni aspetri della politica mediterranea di Giacomo II d'Aragona alla fine del suo regno, in "Sardegna Mediterrunea", Fonti e Studi del Corpus Membrananum Italicarum, prima serie, XXIII, Roma 1983, pp. 101-165; R. TURTAS, La Chiesa durante il periodo aragonese, in Storia dei Sardi e della Sardegna, vol.II, Il Medioevo. Dai giudicati agli aragonesi, Milano 1987, pp. 279-299. L'importanza attribuita dai sovrani calalani ai rapporti con il clero é dimostrata anche dal fatto che il primo registro di Cancelleria di Giacomo II dedicato alla Sardegna si apra proprio con un elenco delle diocesi della Sardegna e della Corsica, Archivio della Corona d'Aragona, Cancelleria (in seguito abbreviato ACA, Canc.)., reg. 341, f. 1 .
} 
equilibrio venutosi a creare dopo la fine delle ostilità, confermarono ai sovrani iberici la necessità di consolidare il potere regio anche attraverso un attento controllo sul clero secolare e regolare, in gran parte di estrazione italiana, possibile in primo luogo caldeggiando presso la Curia pontificia l'immissione, nelle sue file, di religiosi catalani e aragonesi ad essi favorevoli ${ }^{2}$.

L'opportunità di una politica di catalanizzazione del clero sardo, che garantisse l'appoggio delle gerarchie ecclesiastiche alla Corona, veniva sostenuta anche dal cardinale Napoleone Orsini il quale consigliava nel 1325 a Giacomo II che "...hi faça hom son poder, quen les hiles aya bisbes de vostra senyoria...quels prelats que be e mal saben tractar con se volen"3. Lo sforzo dei sovrani catalano-aragonesi fu rivolto, innanzittutto, a porre personaggi di provata fedeltà a capo degli arcivescovadi e vescovadi piu importanti, tentativo che dovette non di rado misurarsi con la volontà centralizzatrice della Santa Sede tendente, in più di un'occasione, a non tenere conto né dei desiderata regi né della tradizionale autonomia dei capitoli cattedralizi ${ }^{4}$.

Accanto al problema, di primaria importanza, dei rapporti con i vescovi, si presentava alla Corona il problema dei rapporti con il clero regolare dell'isola, rappresentato dagli Ordini Mendicanti, principalmente Francescani e Domenicani, che rivestivano un ruolo particolarmente attivo e importante nella società cittadina ${ }^{5}$, e da una serie di ordini monastici di

\footnotetext{
${ }^{2}$ Sulle diverse fasi della guerra di conquista e i rapporti tra la Corona d'Aragona e le entita politiche esistenti nell'isola F.C. CASULA, La Sardegna aragonese, 2 voll., Sassari 1990; B. ANATRA, La Sardegna dall 'unificazione aragonese ai Savoia, Torino 1987.

${ }^{3} \mathrm{H}$. FINKE, Acta Aragonensia, Berlin-Leipzig 1908, doc. 506, pp. 810-814.

'P. MARTINI, Storia ecclesiastica di Sardegna, vol. II, Cagliari 1840, pp. 167 e s8.; D. FILJA, La Sardegna cristiana. Storia della Chiesa, Sassari 1913, vol. II, p. 159 e s8.; M. TANGHERONI, Vescovi e nomine vescovili in Sardegna (1323-1355) in "Sardegna Mediterranea" cit., pp. 167-208; J. TRENCHS-R. SÁINZ DE LA MAZA, Docuinentos pontificios sobre Cerderta de la epoca de Alfonso el Benigno (1327-1336), Barcelona 1983.

SOltre ai Francescani e ai Domenicani erano presenti nell'isola, in misura nettamente minore, fin dalla prima metà del 300 , anche i Carmelitani e gli Agostiniani, cfr. D. SCANO, Codice Diplomatico delle relazioni fra la Santa Sede e la Sardegne, I, Cagliari 1940, doc. CDXL. Non sono stati per il momento rinvenuti documenti catalano-aragonesi relativi ai rapporti con questi Ordini. Sugli stretti legami tra Ordini Mendicanti e città e sul ruolo da essi avuto in ambito urbano E. GUIDONI, Città e Ordini Mendicanti, in "Quademi medievali", 4, 1977, pp. 69-106; J. LE GOFF, L'iminaginario urbano nell'Italia medievale, in "Storia d'ItaliaAnnali", 5, Torino 1982, pp. 543; F. MASALA, Città $e$ insediainenti Francescani in Sardegna. Note per una ricerca, in "Biblioteca Francescana Sarda", a.Il, 1-2, 1988, pp. 171 187.
} 
ceppo benedettino, stanziati soprattutto nelle campagne del settentrione dell'isola, che avevano ricoperto in passato un importante ruolo economico e sociale ma che attraversavano, già dalla seconda metà del Duecento, una grave crisi.

\section{Gli Ordini Mendicanti}

I Francescani erano presenti nel giudicato di Cagliari sicuramente fin dal 1230, quando venne loro affidata, da parte dell'Opera di Santa Maria di Pisa, la chiesa di S. Maria de Portu Gruttis, nelle vicinanze di Castel di Cagliari6. Successivamente, dopo il 1274, essi si trasferirono in un nuovo convento situato nel borgo cittadino di Stampace ${ }^{7}$. Nel corso del XIII secolo i Frati Minori si stabilirono anche nelle principali città degli altri giudicati: dal 1252 e attestato il convento arborense di Oristano, me ntre alla fine del secolo risale la prima menzione documentaria del convento sassarese di Santa Maria di Bethlem, nel giudicato di Torres o Logudoro'.

Nel 1254 fra Nicolo Fortiguerra, visitatore e riformatore, per conto della Curia pontificia, del clero e dei vescovi di Sardegna e di Corsica, fondo, invece, nell'appendice cagliaritana di Villanova, il primo convento

\footnotetext{
${ }^{6}$ Sulla conformazione politica e territoriale della Sardegna medioevale, divisa fino al XIII secolo, nei quattro regni o giudicati di Cagliari, Arborea, Torres e Gallura, F.C. CASULA, Giudicati e curatorie, in "Atlante della Sardegna", a cura di R. Pracchi e A. Terrosu Asole, fasc. II, Roma 1980; La Storia di Sardegna, Sassari 1992. Sulla presenza francescena a Cagliari, P. MARTINI, Storia ecclesiastica cit., II, pp. 65-66; D. FILA, La Sardegna cristiana cit., pp. 106-109; C.M. DEVILLA, I Frati Minori conventuali in Sardegna, Sessari, 1958, pp. 40 e s8.; F. ARTizZU, L'Opera di Santa Maria di Pisa e la Sardegna, Padova 1974, pp. 77 e 88.; G. GossU, La carta pisana del I marzo 1230, primo documento della presenza francescana in Sardegna, e la chiesa di Santa Maria "de Portu Gruttis", in "Biblioteca francescana sarda", I-1 (1987), pp. 4149.

'In quell'anno $i$ frati acquistarono dal chirurgo maestro Tancredi un terreno sul quale iniziarono subito la nuova costruzione, C.M. DEVILLA, I Frati Minori, cit., pp.221 e ss.; G. MELE, Nuove ricerche sui manoscritti liturgici Francescani in Sardegna. Osservazioni su alcuni frammenti neumati clariani (secc.XIII/XIV), in "Biblioteca francescana sarda", II-1/2 (1988), pp.109-135. nt.16.

'C. M. DEVILA, I Frati Minori cit., pp.262 as; G. MELE, Nuove ricerche cit., p.114 e

'Secondo notizie non confortate da alcun documento i Francescani sarebbero stati presenti a Sassari fin dal 1220-25 C.M. DEvilla, Santa Maria di Sassari, Sassari 1961; I Frati Minori cit. pp.251 e 88.
} 
domenicano, per circa trecento anni unico in Sardegna di quest'Ordine ${ }^{10}$. Sia i Frati Minori che i Predicatori dipendevano dalla Provincia di Toscana e mantenevano stretti contatti con le istituzioni religiose pisane che inviavano di frequente loro religiosi nei conventi sardi ${ }^{11}$ I sovrani catalano-aragonesi attribuivano grande importanza a questi ordini religiosi che, attraverso la predicazione e il contatto con il popolo, potevano considerarsi importanti veicoli di propaganda politica e validi strumenti per il consolidamento del potere regio e la pacificazione di territori appena conquistati ${ }^{12}$. I Francescani, in particolare, godevano di grande considerazione ed erano tradizionalmente favoriti dai monarchi catalani che se ne servivano spesso sia per motivi religiosi che per lo svolgimento di missioni politiche e diplomatiche $^{13}$. L' esigenza di poter contare anche in Sardegna su Francescani fedeli alla Corona é dimostrata dal proposito espresso, già nel 1325, dall'infante Alfonso al fratello Pietro, di non permettere il ritorno in Catalogna dei religiosi giunti al seguito delle armate regie e di inviare nei territori recentemente acquisiti altri fratres naturales terre nostre perché potessero popolare un monastero di nuova fondazione ${ }^{14}$. Un nuovo convento francescano

${ }^{10}$ D. FIUA, La Sardegna cristiana cit., p.116 e 8s. Per la storia dei Domenicani in Sardegna é di grande interesse la tesi di laurea, inedita, di G. MELAS, I Domenicani in Sardegna, a.a.1933-34 preziosa per la mancanza di studi sulla storia di quest'ordine nell'isola, cfr. R. PORRA', Gli Ordini Mendicanti in Sardegna durante l'epoca catalano-aragonese. Rassegna bibliografica, in "Bollettino bibliografico della Sardegna", IV-8 (1987), pp.43-50.

"Strettissime erano, per esempio, le relazioni tra il convento domenicano di Cagliari e quello, del medesimo Ordine, di Santa Caterina di Pisa, dal quale uscirono, tra l'altro, molti vescovi che ressero alcune tra le più importanti diocesi sarde, cfr. G. MELAS, I Domenicani cit.

12J. WeBsTER, The early catalan mendicants in Sardinia, in "Biblioteca Francescana Sarda", a.II, 1988, nn. 1-2, pp. 1-14. Sul ruolo degli Ordini Mendicanti come garanti della pace sociale e come strumento di controllo di massa cfr. E. GuIDONI, Ciıld e Ordini Mendicanti cit. Sui Frati Minori in Catalogna e i loro rapporti con i sovrani catalano-aragonesi cfr. J. WEBSTER, Los primeros ficiles inenores en Cataluña, in "Actas del X Congreso de Historia de la Corona de Aragón", vol. 3, 4 y 5, pp. 269-277, Zaragoza 1980; La importancia socio-politica de los frailes menores en la Corona de Aragón hasta el compromiso de Caspe, in "Actas del VI Congreso de la Asociación Intemacional de Hispanistas", Toronto 1977; Ejemplos de devoción real a los franciscanos de Valencia. Piedad o politica?, in "Atti dell'XI Congresso di Storia della Corona d'Aragona", vol. 4, pp. 443-49, Palermo 1984.

${ }^{13}$ Anche l'infante Alfonso, durante il suo soggiomo in Sardegna per la campagna militare di conquista, si avvalse dell'opera dei Frati Minori: per le sue esigenze spirituali chiese infatti dall'assedio di Iglesias l'invio dalla Catalogna di tre religiosi dell'ordine di San Francesco, uno dei quali avrebbe dovuto essere il suo confessore, e francescano era pure un frate che venne inviato con dei messaggi al giudice d'Arborea Ugone II durante l'assedio di Castel di Cagliari nel maggio del 1324, ACA, Canc., reg. 396, f. 52v.; reg. 397, f. 170.

14J. WEBSTER, The early catalan mendicants cit., doc. 1 in Appendice. 
venne fondato, infatti, a Villa di Chiesa per iniziativa dell'Infante, che donò ai frati, nel settembre del 1326, un appezzamento di terreno all'interno delle mura della citta e li sostenne costantemente con aiuti di vario genere $^{15}$.

La presenza nell'isola, e in particolare a Cagliari, nel convento di San Francesco di Stampace e in quello domenicano di Villanova, di un gran numero di religiosi pisani, poteva costituire un elemento di instabilità nel delicato equilibrio venutosi a creare sia dopo la fine della prima fase della guerra con Pisa (giugno 1324), che dopo la conclusione del conflitto. La partecipazione dei frati alla vita pubblica della citta, i legami con il ceto mercantile, il ruolo anche economico svolto soprattutto dal fiorente convento stampacino, erano l'espressione della profonda compenetrazione dei religiosi con la popolazione e con la classe dirigente pisana di Castel di Cagliari. Già l'ammiraglio regio Francesc Carroz e il capitano di guerra Ramon de Peralta, durante il secondo conflitto con il comune toscano, avevano individuato il problema e, nel corso del saccheggio operato dai Catalani nel borgo di Stampace, avevano ordinato la distruzione della ricca chiesa dei Frati Minori sostenendo l'opportunita "que d'aqui avant no hi esteguessen mas frares catalans, e que fossen provencia per ells mateix, e que aixi mateix fossen catalans de totes les ordens qui serien en Sardenya e en Corsega"16. Tuttavia, dopo la pace del 1326, in conformità con gli accordi stipulati con i Pisani al momento della resa di Castel di Cagliari, che stabilivano la permanenza nella città dei suoi vecchi abitanti, se non sospetti di cospirare contro il governo aragonese ${ }^{17}$, i frati toscani, sia $\mathrm{Fr}$ ancescani che Domenicani, continuarono a stare nei loro conventi. I problemi di sicurezza posti da questo stato di cose indussero pero molto presto

\footnotetext{
${ }^{15}$ ACA, Canc., reg. 402, f. 177; C.M. DEVII__A, I Frati Minori cit., pp. 271 e ss.; M. TANGHERONI, La città dell'argento, Napoli 1985, p. 266. Tre anni dopo la nuova fondazione era terminata e a sostegno della nuova comunita il sovrano concedeva il vestiario per ogni anno, concessionc che si tramutò più tardi in una sovvenzione finanziaria percepita sempre con grande difficoltà, vedi nota 36.

${ }^{16}$ Cronica de Ramon Muntaner, in "Les quatre grans cròniques" a cura di F. SOLDEVILA, Barcelona 1971, cap. CCLXXXVII, p.929

${ }^{17} \mathrm{P}$. TOLA, Codex Diplomaticus Sardiniae, tomo I, parte II, Torino 1861 (rist. anast. Sassari 1984), doc. XXXII, pp. 677-681; A. ARRJBAS PALAU, La conquista de Cerderia por Jaime II de Aragón, Barcelona 1952, doc. LVII, pp. 445447; F.C. CASULA, I trattati diplomatici sardo-aragonesi del 1323-1326, in "Sardegna, Mediterraneo e Atlantico tra Medioevo ed Età Modema. Studi storici in memoria di Alberto Boscolo", vol. I, Roma 1993, pp. 207-220.
} 
l'Infante a decidere l'espulsione massiccia dei Pisani sulla base di vera o presunta attività antiaragonese ${ }^{18}$. Anche sui Frati Minori e Predicatori si addensarono sospetti di trame eversive, come si apprende da una lettera inviata il 1 gennaio 1327 a Giacomo II dall'infante Alfonso il quale sosteneva la necessità di allontanare dalla città e da Bonaria i religiosi toscani di intrambi gli ordini ${ }^{19}$. Precise istruzioni in questo senso vennero inviate al governatore Boxadors tramite l'ambasciatore regio Ramon Sa-Vall: "Primerament que.l senyor infant ha entes per alguns amichs seus qui li.n han fet saber, que.ls Frares Menors e Preycadors de Pisa qui trien en castell de Caller, tracten ço que poden en perill del Castell e en deservey del senyor Infant, per que vol lo senyor Infant que Frares Menors ne Preycadors pisans no aturen aqui, e aço sia presa com millor manera puga"20. Anche nella successiva corrispondenza con il governatore del regno di Sardegna l'Infante ribadiva "que no vol que negun religios de nacio pisana atur en Caller ne en los monestir d'aquella"21. L'occasione per poter esercitare un maggiore controllo sugli Ordini Mendicanti- che rimanevano strettamente legati alla penisola italiana attraverso la dipendenza dai superiori toscani- e per dare un più forte impulso alla catalanizzazione del clero regolare e secolare, venne con lo scisma provocato dall'imperatore Ludovico il Bavaro che, in lotta con il pontefice Giovanni XXII, nel maggio del 1328 aveva eletto papa, con il nome di Niccolo V, un frate minore, Pietro da Corvara. A Pisa, dove avevano soggiornato diverso tempo, l'imperatore e l'antipapa godevano di un certo seguito tra il clero, e soprattutto tra i Francescani

\footnotetext{
${ }^{18}$ Sull'espulsione dei pisani dal Castello di Cagliari e sul ripopolamento della città con catalano-aragonesi R. CONDE I DELGADO DE MOLINA-A.M. ARAGO CABAÑAS, Castell de Caller. Cagliari catalano-aragonese, Cagliari 1984.

${ }^{19} \mathrm{ACA}$, Canc., reg. 410, f. 119; J. WEBSTER, The early catalan mendicants cit., doc. 2 in Appendice.

${ }^{20}$ R. CONDE-A.M. ARAGÓ, Castell de Căller cit., Apendice Documental, doc. IV, p. 216. Cfr. anche J. ZURITA, Anales de la Corona de Aragon, libro VII, cap. VI, p. 319, Saragozza 1972. "...se tuvo aviso que los frailes de la orden de predicadores y de los menores de Caller, que eran pisanos, tentaban de entregar el Castillo de Caller a los de Pisa y estuviese aquella fuerza en poder de aquella señoria, mandose que todos saliesen de Caller" e G.F. FARA, De rebus Sardois, libro III, ed. a cura di Enzo Cadoni, p. 49, Sassari 1992.

${ }^{21}$ R. CONDE-A.M. ARAGO, Castell de Caller cit., Apendice Documental, doc. V, p. 221, doc. VI, p. 224. Anche dopo l'occupazione di Alghero, avvenuta nel novembre del 1354, si presenterà lo stesso probleına riguardo ai religiosi stranieri: nel febbraio del 1355 Pietro IV stabiliva che "...fratres aliqui de ordine minoruın, nec alterius cuiuscumque ordinis, nisi sint Cathalani, vel Aragonenses, non audeant seu presumant in dicta villa habitationem nec incolatum quocumque modo facere...", P. TOLA, Codex cit., t.I, p.II, doc. XCIX, p. 767.
} 
seguaci della dottrina pauperistica propugnata dal deposto ministro generale Michele da Cesena e condannata come eretica da Giovanni XXII. Attraverso i Frati Minori pisani che stavano in Sardegna ed altri inviati appositamente dalla città toscana a scopi propagandistici, queste teorie scismatiche ed eretiche ebbero una certa diffusione nei conventi dell'isola, dove trovarono rifugio e protezione alcuni frati seguaci di Michele da Cesena ${ }^{22}$. Numerosi documenti dell'anno 1329 attestano i tentativi compiuti dalla Corona per incrementare la presenza catalana nelle file del clero sardo e per allontanare quanto più possibile dall'isola $\mathrm{i}$ religiosi pisani approfittando del seguito di cui l'antipapa godeva presso il clero del Comune toscano: é particolarmente significativo, a questo proposito, il memoriale inviato al papa Giovanni XXII da Alfonso il Benigno tramite il suo procuratore presso la curia pontificia Gondisalvo Çapata, arcidiacono di Tarazona, il 10 aprile di quell'anno. Il sovrano informava il pontefice della presenza nell'isola di numerosi ecclesiastici pisani, seguaci di Ludovico il Bavaro e dell'antipapa scismatico, infiltratisi clandestinamente, molti dei quali erano stati espulsi dal governatore generale perchè "palam et publice in aliquibus locis nitebantur falsas et venenosas informaciones inter gentes illarum partium seminare, illum malditum antipapam et eciam Lodovicum de Bavaria, qui se imperatorem nominat, diversimode commendando"; sottolineava poi la pericolosità costituita dalla soggezione di cattedrali, chiese e ordini religiosi alle autorità religiose pisane cosicchè "episcopi et rectores et fratres eorum tam dicto domino pape quam dicto domino regi sunt plurimum suspecti et periculosi", e chiedeva che venissero recisi i legami di queste istituzioni con il Comune toscano, che le chiese dipendessero dagli arcivescovadi dell'isola e che gli ordini religiosi venissero uniti alla provincia di Aragona

\footnotetext{
${ }^{22}$ Le prime avvisaglie dello scisına si ebbero nella zona di Sassari gia nel 1328, come si evince da due lettere del papa Giovanni XXII inviate all'arcivescovo d'Arborea Guido Cattaneo, inquisitore "hereticae pravitatis in Regno Sardiniae", D. SCANO, Codice Diplomatico cit., vol. I, docc. CCCLXXXVI, CCCLXXXVII; J. TRENCHS-R. SAINZ DE LA MAZA, Documentos pontificios cit., docc. 26, 27. Sulla figura di Guido Cattaneo O. SCHENA, Una presenza sarda al convegno di Avignone del 1322 sulla povertà evangelica, in "Clio", a.XV, n.1, 1979, pp.139-175. Ancora nel 1333 il Cattaneo si accaniva in un processo contro alcuni frati considerati eretici tanto da suscitare l'intervento papale in favore degli accusati, $D$. SCANO, Codice Diplomatico cit. doc. CDXXVI; J. TRENChS-R. SÁINZ DE LA MAZA, Documentos pontificios cit., doc.235. Sui riflessi dello scisma di Pietro da Corvara in Sardegna si vedano anche, in quest'ultimo volume, $i$ docc. $24,25,233,234$, nonché $M$. TANGHERONI, Vescovi e nomine cit., pp. $186-191$.
} 
e Catalogna ${ }^{23}$. Giovanni XXIl accolse l'appello del sovrano che, nel giugno del ' 29 riusciva ad ottenere che i Francescani e i Domenicani dell'isola venissero separati dalla Provincia di Toscana e passassero a dipendere dalla Provincia d'Aragona ${ }^{24}$. Nel 1329 veniva nominato anche il primo vicario generale catalano per i Frati Minori della Sardegna, frate Guerau de Prat, che Alfonso metteva sotto la sua protezione insieme a tutti i conventi sardi dell'Ordine $^{25}$. Il sovrano chiedeva anche al pontefice che al vicario fosse attribuito l'officium inquisicionis, tradizionalmente esercitato dai Frati Minori della Toscana ${ }^{26}$.

\footnotetext{
${ }^{23}$ ACA, Canc. reg. 508, ff. 240-24lv. Nell'aprile del 1329 il sovrano chiese anche al pontefice di rendere indipendente l'ospedale di Santa Lucia di Villa di Chiesa dall'Ospedale Nuovo della Misericordia di Pisa, al quale cra soltoposto, in conseguenza dei contrasti sorti tra il rettore designato dal governo aragonese, il valenzano fra' Martino de Cabans, e quello scelto da Pisa, fra' Runicri, accusato di essere un seguace di Ludovico il Bavaro e dell'antipapa scismatico, ACA, Canc., reg. 509, f.4; M. TANGHERONI, La città cit., pp.6364. dal quale risulta pure che l'appcllo di Alfonso non venne accolto. Lo stesso studioso, Note sui rapporti tra Pisa, l'Aragona e Genova all'epoca di Alfonso il Benigno, in "Alti del I Congresso Storico Liguria-Catalogna", Bordighera 1974, pp. 177-182, afferna che il sovrano aragonese vide nello scisma l'occasione per eliminare definitivamente i Pisani dalla Sardegna con l'incameramento dei beni che erano loro rimasti nell'isola, ma la fine dello scisma nel giugno del 1329 impedi ad Alfonso il conseguimento dei suoi obiettivi. Sempre nel 1329 il sovrano ribadiva al cardinale Bertrando la richiesta, già avanzata precedentemente, di promuovere l'invio nel regno di Sardegna di religiosi catalani e aragonesi, ACA, Canc., reg. $509, \mathrm{ff} .4 \mathrm{v}-5$. Per un maggior impulso alla catalanizzazione del clero il Benigno contava anche su Guido Cattaneo, arcivescovo d'Arborea e uno dei più influenti prelati dell'isola, con il quale lamentava gli scarsi risultati fino a quel momento raggiunti in questa impresa, ACA, Canc, reg. 509, f. 3 del 19 aprile 1329 regestato da A. BOSCOLO in Documenti sull'economia e sulla societa in Sardegna all epoca di Alfonso il Benigno, Padova 1973, doc.62, pp.17-18; efr. anche M. TANGHI:RONI, Vescovi e nomine cit., p. 40-41.

${ }^{24}$ D. SCANO, Codice Diplomatico cit., doc. CCCXCI del 30 giugno 1329; D. FILAA, La Sardegna cristiana cit., pp. 168 e ss.; C.M. DEVIL. A, I Frati Minori cit., pp. 93 e ss.; J.R. WEBSTER, The early calalan inendicants cit., pp. 4 e ss.

${ }^{25}$ ACA, Canc., reg. 509, ff. 63-63v.; J.R. WEBSTER, The early catalan mendicants, cit., p. 4. Alla morte di Gucrau de Prat i consiglicri e probiuomini del Castello di Cagliari suggerirono al re di appoggiare la nomina di fra' Pictro Ivern, presumibilmente catalano, "esperto in scrinoni come in altri salutari rimedi delle anime del popolo", F.C. CASUIA, Carte Reali Diplomatiche di Alfonso III il Benigno, re d'Aragona, riguardanti l'llalia, Padova 1970., doc. 348, p. 206; anche il giudice d'Arborea Ugone II, nel 1331, riteneva necessaria la nomina di un vicario generale aragonese o catalano, bidern, doc. 117, p. 117 . Nello stesso anno venne nominato all'importante carica frate Bartolomeo Peregrini, che il sovrano raccomandava al govematore dell'isola Raimondo de Cardona, ACA, Canc., reg. 512, ff. 248v-249).

${ }^{26}$ ACA, Canc., reg. 510, f. 188; C.M. DeVIl.LA, I Frati Minori cit., pp. 52 e ss. In occasione di una controversia nata nel 1362 tra i Domenicani e i Francescani per l'esercizio dell'inquisizione nell'isola, il sovrano Pictro IV confermava questo diritlo al vicario dei Frati Minori, che aveva sempre esereitato "dictam inquisicionem heretice pravitatis in partibus ytalie, ad cuius instar dicta insula populata existit", ACA, Canc., reg. 1035, if. 21v.-22.
} 
Contemporaneamente iniziava anche la catalanizzazione dell'ordine dei Predicatori. I Domenicani toscani erano presenti in numero consistente nel convento cagliaritano di Villanova che aveva sempre mantenuto stretti legami con il convento pisano di Santa Caterina. Dopo il passaggio dell'Ordine alla Provincia d'Aragona e Catalogna, considerando anche i sospetti di trame filopisane che erano caduti, oltre che sui Francescani, anche sui Domenicani, la sostituzione dei frati pisani con elementi iberici si rese inderogabile. Motivando la richiesta con la preoccupazione per la salute spirituale dei suoi sudditi catalani di Cagliari, che si trovavano in difficolta nelle confessioni e nell'intendere i sermoni espressi in una lingua diversa dalla loro, Alfonso chiese più volte ai superiori aragonesi l'invio nel convento cagliaritano, considerato caput et fundamentum dell'Ordine nell'isola, di un discreto numero di Frati Predicatori provenienti dagli stati continentali della Corona che fossero in grado di prestare la necessaria assistenza religiosa agli abitanti della citta ${ }^{27}$.

Un ulteriore tentativo per rafforzare la presenza catalana all'interno del clero sardo fu l'introduzione nell'isola dell'Ordine della Merce che era nato a Barcellona nella prima metà del XIII secolo e che era largamente diffuso negli stati della Confederazione ${ }^{28}$. Ai Mercedari Alfonso il Benigno concesse il 17 ottobre 1335 il patronato sulla chiesa di Santa Maria di Bonaria ma, per la morte del sovrano, avvenuta pochi mesi dopo, la concessione non ebbe effetto. Venne rinnovata il 15 giugno 1336 da Pietro IV; l'insediamento dei Mercedari venne pero ostacolato dalle diatribe con l'arcivescovo di Cagliari che rivendicava il patronato sulla chiesa. In effetti non si hanno notizie sulla presenza di questi religiosi a Cagliari almeno fino al $1397^{29}$.

\footnotetext{
${ }^{27}$ ACA, Canc., reg. 410, f. 119; reg. 509, ff. 4-4v. reg. 512, f. 286. La fiducia attribuita dalla Corona a meinbri iberici degli Ordini Mendicanti come veicoli per la catalanizzazione dei territori sardi conquistati, é evidente anche nella volontà di Alfonso il Benigno di assegnare alcuni vescovadi vacanti a frati Francescani e Domenicani catalano-aragonesi: nel 1329 Alfonso otteneva dal Papa il vescovado di Galtellì per il frate predicatore Gualterio de Aydona, ACA, Canc., reg. 509, ff. 60-60v; per lo stesso vescovado, resosi nuovamente vacante, il sovruno chiedeva qualche anno dopo la nomina di frate Francesc Gosalt, dell'Ordine dei Minori, ACA, Canc. reg. 515, ff. 45-45 v.,dell'ottobre 1332/giugno 1333; sempre nel 1333 il sovrano otteneva dal Pontefice il vescovado di Ampurias per il suo "natural" Jauine de Prat, dell'Ordine dei Predicalori, ACA, Canc., reg. 515, f.50.

28J.W. Brodman, L'Orde de la Mercè, Barcelona 1990.

${ }^{29}$ M.M. CosTA, El Santuari de Bonaire, Cagliari 1973.
} 
Non abbiamo dati sufficienti per stabilire il livello di catalanizzazione raggiunto in questo periodo all'interno degli Ordini Mendicanti, anche se la necessità più volte ribadita dai sovrani, negli anni successivi, di dare un maggior impulso all'inserimento di religiosi iberici nelle file del clero, potrebbe far pensare che, nonostante gli sforzi, l'operazione non avesse raggiunto $\mathrm{i}$ risultati sperati ${ }^{30}$. Forse per questo nel 1456, seppure in un contesto politico ormai completamente diverso rispetto a quello della prima metà del Trecento, considerando anche la grande potenza economica e sociale raggiunta dal convento di San Francesco di Stampace, i consiglieri di Cagliari chiesero al Papa Callisto III che, nel rispetto della volontà dei precedenti sovrani e delle ordinanze da loro emanate, le cariche ecclesiastiche e, specificatamente, quelle dei Frati Minori, venissero affidate soltanto a Catalano-Aragonesi ${ }^{31}$.

L'importanza politica e sociale attribuita dalla Corona agli Ordini Mendicanti, unita alla stima e alla devozione manifestata più volte da Alfonso, soprattutto nei confronti dei Francescani, "...ob reverenciam et devocionem quam ad beatissimum Franciscum et eius ordinem gerimus et habemus...", indussero il sovrano ad occuparsi con sollecitudine anche del problema delle conseguenze della guerra di conquista sui conventi situati nelle appendici del Castello di Cagliari e a soccorrere generosamente $\mathbf{i}$ religiosi nelle loro necessità materiali. Molti sono i documenti che riportano le lamentele dei frati per gli ingenti danni subiti e per lo stato di estrema indigenza cui si dicevano ridotti. La chiesa francescana di Stampace, qui era molt rica ${ }^{32}$, e l'annesso convento dei Frati Minori non solo erano stati semidistrutti, ma avevano subito gravi ruberie sia da parte dei Pisani che da parte dei Catalani che li avevano spogliati di suppellettili, paramenti, libri ed altri beni. La stessa sorte aveva subito il monastero di Santa Margherita, situato sempre nell'appendice di Stampace, nel quale risiedevano le mona-

\footnotetext{
${ }^{30}$ La politica di catalanizzazionc del clero incontrò non poche difficoltà come si evince anche da un documento emanato da Pietro IV nel 1342, quasi vent'anni dopo l'arrivo dei Catalano-Arngonesi in Sardegna, nel quale il sovrano ribadisce la necessità che gli incarichi ecclesiastici venissero assegnati solo a persone di fiducia, specialmente aragonesi, valenzani e catalani, cosa che non sempre avveniva, L. D'ARIENZO, Carte Reali Diplomatiche di Pietro IV il Cerimonioso, re d'Aragona, riguardanti l'Italia, Padova 1971, doc.156, p.71.

${ }^{31}$ D. SCANO, Codice Diplomatico cit., vol. II, doc. CLX. Per la grande importanza del convento di San Francesco di Stanpace nella società cagliaritana del XV secolo G. OuA REPETTO, La società cagliaritana nel 400 in "Cultura quattro-cinquecentesca in Sardegna. Retabli restaurati e documenti" (cutalogo della mostra), pp. 19-24.

${ }^{32}$ R. Muntaner, Crònica cit., cap. 287, p. 52.
} 
che dell'ordine di Santa Chiara e presso il quale i frati avevano trasportato molti beni di loro proprietà nella vana speranza di metterli in salvo. L'avidità rapace delle soldatesche di entrambe le parti non aveva avuto rispetto neppure per la clausura vigente nel convento clariano e tutti $i$ beni in esso custoditi, appartenenti alle stesse monache e ai Francescani, erano stati rubati $^{33}$. Grande povertà lamentavano anche i Frati Minori di Villa di Chiesa e quelli di Sassari ${ }^{34}$. Nei confronti di tutti questi conventi Alfonso intervenne generosamente: ai Francescani di Cagliari fu concessa una somma di 500 lire di alfonsini minuti perché potessero riparare il monastero e la chiesa, ridotta in condizioni tali che non vi si potevano neppure celebrare le sacre funzioni ${ }^{35}$; a quelli di Villa di Chiesa veniva assicurato il vestiario per ogni anno, concessione tramutata poi in una sovvenzione di venticinque lire di alfonsini minuti annuali ${ }^{36}$; la stessa somma veniva concessa ai Frati Minori di Sassari ${ }^{37}$ mentre alle Clarisse di Stampace avrebbero dovuto andare 200 starelli di grano all'anno a misura di Cagliari da trarre dai proventi della dogana, oltre alla restituzione dei beni occupati durante la guerra ${ }^{38}$. Ma la constatazione che le rendite dell'isola non erano quelle sperate e le difficoltà tinanziarie che subito i Catalani dovettero affontare a causa delle ingenti spese militari, fecero sl che gli aiuti promessi ai religiosi restassero per lungo tempo solo sulla carta ${ }^{39}$. Per venire

\footnotetext{
${ }^{33} \mathrm{ACA}$, reg. 402 , ff. $175 \mathrm{v}-176$ e $176 \mathrm{v} .-177$ del 22 e 23 settembre 1326. Sono questi i primi documenti che fanno riferimento alle Clarisse del monastero di Santa Margherita di Cagliari, cfr. M.G. MELONI, Note sulla presenza delle Clarisse in Sardegna nel Medioevo, in "Bollettino Bibliografico e Rassegna Archivistica di Studi Storici della Sardegna", XI-18 (1994), pp. 43-52.

${ }^{34}$ Data la grande povertà dei conventi dei Frati Minori, Alfonso ordinava al govematore Bemardo de Boxadors di indurre tutti i debitori a pagare loro le somme dovute, F.C. CASULA, Carte Reali cit., doc. 41, p. 74.

${ }^{35}$ ACA, Canc., reg. 402, ff. $175 v .-176$ e 176v.-177 del settembre 1326. 1331 .

${ }^{36}$ ACA, reg. 508, ff. 237v.-238 del 4 aprile 1329; reg. 512, ff. 161-16/v. del 15 luglio

${ }^{37} \mathrm{ACA}$, reg. 508 , f. $238 \mathrm{v}$. del 4 aprile 1329. I frati sassaresi lamentavano la pendita, dopo il passaggio della citta agli aragonesi, del sussidio di 25 lire di monete genovine che ricevevano dal precedente govemo, e Alfonso concesse loro, in sostituzione, un sussidio di 25 lire annuali di alfonsini minuti.

${ }^{38} \mathrm{ACA}$, reg. 40, ff. 175v.-177; reg. 508, ff. 75-76.

${ }^{39} \mathrm{Ciò} \mathrm{si} \mathrm{deduce} \mathrm{dai} \mathrm{numcrosi} \mathrm{documenti} \mathrm{che} \mathrm{riportano} \mathrm{le} \mathrm{lagnanze} \mathrm{dei} \mathrm{religiosi} \mathrm{per} \mathrm{la}$ mancata upplicazione dei provvedimenti regi e la conferma degli stessi da parte dei sovrani che si succedettero sul trono, ACA, Canc., reg. 403, f. 55; reg. 508, f. 238; reg. 510, ff. 186v.187; reg. 514, f. 244; C.R.D. di Giacomo II, c. 9728, cassa 79; reg. 1009, f. 265 per quanto riguarda il convento di San Fruncesco di Stampace; reg. 512, ff. 154-154v.; reg. 514, ff.
} 
incontro alle esigenze dei Frati Minori, che in molti casi erano costretti a rinunciare a lasciti testamentari impugnati dai parenti del defunto, per il divieto loro fatto di comparire in giudizio, Alfonso stabiliva, inoltre, la nomina di speciali curatori incaricati di rappresentare i religiosi in giudizio e di riscuotere a loro nome elemosinas et legata ${ }^{40}$.

Il favore di Alfonso verso i Francescani è dimostrato anche dal sostegno che questi ottennero dal sovrano nelle numerose controversie sorte con le autorità diocesane e con il clero secolare. L'antagonismo tra il clero secolare e gli Ordini Mendicanti, fatto annoso e generalizzato, nasceva dagli amplissimi privilegi relativi alla cura animarum concessi ai Mendicanti dal pontefice Martino IV, privilegi di cui i frati continuavano ad usufruire nonostante le limitazioni a favore del clero parrocchiale imposte da una bolla di Bonifacio VIII ${ }^{41}$. Dapertutto i frati erano riusciti a guadagnarsi la devozione e l'affetto delle popolazioni che correvano ad ascoltare i loro sermoni, si affollavano intorno ai loro confessionali, sceglievano di farsi seppell ire nei loro cimiteri. Tutto ciò non poteva non suscitare l'ostilità del clero parrocchiale che si vedeva spodestato nelle sue funzioni, dalle quali traeva la maggior parte della sua influenza e delle sue rendite. Alfonso il Benigno, come si é detto, prese decisamente le difese dei Francescani nelle controversie sorte tra questi e il clero di alcune città dell'isola. Negli anni 1329-1330 egli intervenne a favore dei Frati Minori di Oristano angariati dall'arcivescovo Guido Cattaneo il quale addirittura negava il sacramento

149v.-150; reg. 1009, f. 265v.; reg.1018, f.39; reg. 1030, ff.103v.-104 per le monache di Santa Chiara di Cagliari; reg. 510, ff. 187-187v., L.D'AkIrnz.O, Carte Reali cit., doc.409, pp. 207-208 per i Frati Minori di Villa di Chiesa; reg. 1007, f. 245, emanato da Pietro IV il 13 agosto 1337, per i conventi di Cagliari, Sassari e Villa di Chiesa. Concessioni di grano sardo vennero fatte da Alfonso anche a numerosi monasteri catalani, ma anche queste non vennero quasi mai osservule: 100 starelli all'anno al monastero cistereense di Valldonzella (reg. 401 , f. 122; reg. 418, ff. 132v.-133; reg. 1020, ff. 38-38v.); a quello di Jonqueres (reg. 403, ff. 97v.-98); alle ininorisse di Santa Chiara di Lerida (reg. 401, f. 118, I agosto 1326); al convento dei frati Predicatori di Barcellona (reg. 401, f.97 reg. 1009, ff. 212 v.-213). Al monastero delle Clarisse di Pedralbes, di recente fondazione, vennero invece assegnati 4000 soldi all'anno da trarre dalle dogane di Cagliari (reg. 403, ff. 95v.-96v.). Sull'invio di grano sando in Catalogna, dovuto alla penuria di questo prodotto durante il regno di Alfonso il Benigno, cfr. J. MUTGE VIVES, Trigo sardo en Barcelona durante el reinado de Alfonso el Benigno, in "VIII Congreso de Historia de la Corona de Aragón", II, 3, Valencia 1973, pp. $235-41$.

${ }^{40} \mathrm{ACA}$, Canc., reg. 510, f. 187v.

"La bolla è la "Super Cathedram" del 18 fcbbraio del 1300, Bullarium Franciscanum Romanoruin Pontificuin, tomo IV, Roma 1768, rist. anast., Assisi 1984, doc. CLXXIX, Pp. 498-500; le disposizioni in essa contenute furono in seguito attenuate e nuovamente ripristinate dai successori di Bonifacio. 
dell'eucarestia a coloro che si fossero confessati con $\mathrm{i}$ frati $\mathrm{o}$ avessero deciso di farsi seppellire presso di loro $0^{42}$. Ancora più ferma fu la posizione del sovrano in un analogo conflitto, scoppiato a Cagliari nel 1333, che vide contrapposti i Frati Minori e le monache Clarisse, da un lato, e l'Arcivescovo catalano della stessa città, Gondisalvo, dall'altro ${ }^{43}$. L'Arcivescovo contestava, sia in prima persona che attraverso il clero delle parrocchie, il diritto dei Francescani di seppellire nella loro chiesa i fedeli che avessero espresso questo desiderio o che avessero una tale tradizione familiare. Quest'uso era frequentissimo a Cagliari per la grande popolarità e devozione di cui erano oggetto i Francescani: sia membri delle famiglie nobili, alcune delle quali avevano una cappella nella chiesa di San Francesco, che molti popolani sceglievano di essere sepolti presso i Frati Minori e questo privava il clero parrocchiale di una grossa fetta dei proventi derivanti dai funerali ${ }^{44}$. Da qui le molestie lamentate dai frati che dovettero ricorrere al sovrano perché venissero rispettati i loro diritti. L'Arcivescovo, inoltre, violava i privilegi delle Clarisse molestandole con visite indebite e inopportune che, secondo le disposizioni papali, dovevano essere effettuate solo dai Francescani, tanto da costringere le monache a denunciare il fatto alla Curia Romana. Alfonso prese decisamente le difese dei religiosi ingiungendo all'Arcivescovo di smettere di molestarli e di andare contro le antiche usanze e i loro privilegi. Poco fiducioso in un ravvedimento dell'alto prelato, si rivolgeva anche al governatore Raimondo de Cardona perché tenesse sotto controllo la situazione e non permettesse che i diritti dei frati e delle monache venissero calpestati ${ }^{45}$. Un anno dopo il comportamento dell'Arci-

${ }^{42}$ ACA, Canc., reg. 508, ff. 235-237; reg. 510, ff. 209-210. In questi ultimi documenti il sovrano prende anche le difese del Terz'Ordine francescano, anch'esso duramente avversato dal Cattaneo. Lo stesso arcivescovo d'Arborea, pur così ostile ai Francescani, apparteneva all'Ordine dei Minori, efr. O. SCHENA, Una presenza sarda cit., pp. 139-140.

${ }^{43}$ Sulla figura dell'arcivescovo Gondisalvo, che resse la diocesi cagliaritana dal 1331 al 1340 M. TANGHERONI, Vescovi e nomine cit., pp. 25 e ss.

${ }^{4}$ Per una descrizione artistica e architettonica della chiesá di San Francesco di Stampace, crollata nel 1875, e delle lastre tombali che erano murate nelle pareti e nel pavimento $D$. SCANO, Storia dell'arte in Sardegna dal XI al XIV secolo, Bologna 1979 (rist. anast. dell'edizione del 1907), pp. 396 s8.; sulle tombe che si trovavano al suo intemo anche C.M. DEVuLA, I Frati Minori cit., pp. 226 ss.; R. PORRA', Le sepolture a San Francesco di Stampace, in San Francesco di Stampace. I perchè del recupero, Cagliari 1986, pp. 17-20; M.M. CosTA, Les sepultures de la fainilia Carrds en el monestir de Sant Francesc de Caller, in "Biblioteca francescana sarda", I-1 (1987), pp. 1-39.

${ }^{45}$ ACA, Canc., reg. 516, ff. 137-137 v. del 16 luglio 1333; J.WEBSTER, The early catalan. mendicants; cit, pp. 5-6. 
vescovo non era mutato: non solo cercava di distogliere i fedeli dal farsi seppellire nel cimitero francescano "inhibendo ne impuberes cum suis patribus in eorum ciminteriis tumulentur", ma era giunto a comminare la scomunica contro i benefattori dei frati e contro coloro che si confessavano da loro senza aver chiesto prima il permesso dei sacerdoti parrocchiali, diffamandoli pubblicamente ut fidelium devotio algeat. Il sovrano era dovuto intervenire nuovamente, dietro richiesta dei Francescani, invitando fermamente l'Arcivescovo a desistere dal suo atteggiamento e a permettere ai religiosi di esercitare i loro diritti per il bene delle anime ${ }^{46}$. Non sembra che gli interventi regi nelle controversie tra i Mendicanti e il clero secolare abbiano avuto grande efficacia se, nel marzo del 1336, dovette intervenire il pontefice Benedetto XII per cercare una soluzione all'annoso proble$\mathrm{ma}^{47}$.

Come si é visto, l'atteggiamento di Alfonso nei confronti degli Ordini Mendicanti fu di grande considerazione, dovuta sia ad autentica devozione sia alla coscienza del loro peso sociale e religioso. La politica attuata nei loro confronti fu dunque improntata sull'esigenza di assicurarsene la fedeltà e l'appoggio, talvolta anche ponendosi contro il clero diocesano, attraverso un attento controllo, ottenuto incentivando la presenza catalana tra i religiosi.

Di diverso aspetto l'atteggiamento del sovrano nei confronti dell'altra branca del clero regolare dell'isola: gli ordini monastici benedettini.

\section{Gli Ordini Monastici Benedettini}

Il monachesimo benedettino si era diffuso nei quattro stati o giudicati in cui era divisa la Sardegna nel Medioevo, tra la seconda metà dell'XI secolo e la prima metà del secolo successivo ${ }^{48}$. Tutte le varie correnti mo-

\footnotetext{
${ }^{40}$ ACA, Canc., reg. 517 ff. 50-51v. del 26 giugno 1334 e f.86v. del 23 ottobre dello stesso anno.

${ }^{47}$ D. SCANo, Codice Diplomatico cit., I, doc. CDXL.

${ }^{48}$ Sull'insediamento dei Benedettini e sui motivi che indussero $i$ giudici e il papato a favorime la diffusione A. BOSCOLO, La Sardegna bizantina e alto-giudicale, Sassari 1982; B. ANATRA, Santa Sede e Sardegna tra Medioevo ed Età Moderna, in "Annali della Facollà di Magistero dell'Università di Cagliari", n.s., vol. IX (1985); G. MrLoNI, La Sardegna e la politica mediterranea di Pisa, Genova e Aragona, in Storia dei Sardi cit., pp. 49-96; Boidem, M. TANGHERONI, L'economia e la società della Sardegna, pp. 157-191; lbidem, G. MiLJA, La
} 
nastiche nate dalla grande famiglia benedettina in Italia e fuori, dai Cassinesi ai Vittorini di Marsiglia, ai Cistercensi, ai Camaldolesi e ai Vallombrosani ebbero in Sardegna loro monasteri che, grazie alla generosità dei sovrani locali e di privati cittadini, poterono godere di vastissime proprietà terriere e di ampi privilegi ${ }^{49}$. La stagione del massimo splendore di queste fondazioni monastiche non ando oltre la prima metà del XIII secolo. Una serie di avvenimenti politici influl negativamente dapprima sulla loro consistenza patrimoniale, poi sulla loro stessa esistenza: l'invadenza pisana, soprattutto nei giudicati di Cagliari e di Gallura, la caduta di tre dei quattro giudicati, che privava i monasteri della protezione e della generosità dei giudici, principali artefici della loro nascita e del loro sviluppo; l'incerta situazione politica determinata da questi avvenimenti particolarmente nel Logudoro, dove era situata la maggior parte dei cenobi, costituirono altrettanti momenti di difficoltà per i monasteri sardi che iniziarono una inesorabile decaden$\mathrm{za}^{\text {so }}$. All'epoca della conquista aragonese alcuni di essi erano già scompar$\mathrm{si}^{\text {s1 }}$; d'altra parte, nel XIV secolo, il monachesimo benedettino era investito, in tutta Europa, da una grave crisi dovuta al mutamento delle condizioni economico-sociali e della sensibilità religiosa che aveva portato all'affermarsi degli Ordini Mendicanti, più vicini alle esigenze dei tempi e alle rinnovate tendenze spirituali dei fedeli ${ }^{\$ 2}$.

La scarsa documentazione relativa a questi monasteri rinvenuta nell'Archivio della Corona d'Aragona rivela che l'interesse della Corona

civiltà giudicale, pp. 193-229; F.C. CASULA, La politica religiosa del giudicato di Torres, in "Rivista cistcrcense", V-1 (1988), pp. 5-10.

${ }^{49}$ Sugli ordini monastici che si stabilirono in Sardegna, A. SABA, Montecassino e la Sardegna, note storiche e codice diploınatico sardo-cassinese, Badia di Montecassino, 1927; A. Boscolo, L'abbazia di San Vittore, Pisa e la Sardegna, Padova 1958; AA.VV., Studi sui Vittorini in Sardegna, Padova 1963; G. ZANEITT, I Vallombrosani in Sardegna, Sassari 1968; IDEM, I Carnaldolesi in Sardegna, Cagliari 1974; IDEM, I Cistercensi in Sardegna e le abbazie di S.Maria di Corte, di Paulis e di Coros, in "Archivio Storico Sardo di Sassari", 2 (1976); AA.VV., I Cistercensi in Sardegna (a cura di G. Spiga), Nuoro 1990.

${ }^{50} \mathrm{Gli}$ avvenimenti politici e militari che si svolsero in Sardegna prima della conquista catalano-aragonese sono delineati da F.C. CASULA, La Storia di Sardegna cit.

${ }^{31}$ Un quadro dei monasteri benedettini ancora esistenti in Sardegna nella prima metà del XIV secolo si puó ricavare dalle Rationes Decimarum Italiae nei secoli XIII-XIV - Sardinia, a cura di P. SELLA, Città del Vaticano 1945, relative agli anni 1342-54.

${ }^{52}$ Sulla crisi del monachesimo tradizionale nei secc. XIII e XIV, G. PENCO, Storia del monachesimo in Italia. Dalle origini alla fine del Medioevo, Milano 1983, p. 253 e 8s.; $R$. MANSELU-E. PASZTOR, Il monachesimo nel basso medioevo, in AA.VV., "Dall'cremo al cenobio. La civiltà monastica in Italia dalle origini all'età di Dante", Milano 1987, pp. 67. 126; M. PACAUT, Monaci e religiosi nel Medioevo, Bologna 1989, p. 217 e 88. 
nei confronti di questi antichi cenobi, la cui importanza religiosa, sociale ed economica era ormai marginale, si appunto tutto sui loro ancor vasti possessi terrieri che vennero incamerati per essere ceduti in feudo ai nobili catalani, aragonesi, valenzani e maiorchini che avevano partecipato all'impresa di conquista.

I primi documenti emanati dalla Cancelleria regia durante la campagna militare per la conquista dei territori pisani della Sardegna, riguardano il monastero vittorino di San Saturno, situato tra il colle sul quale sorgeva il Castello di Cagliari e il colle di Bonaria, sul quale l'infante Alfonso costrul il suo accampamento durante l'assedio della rocca cagliaritana. Il priorato di San Saturno, che durante il periodo pisano aveva subito un notevole ridimensionamento delle sue vaste proprietà terriere, si trovava in quegli anni in uno stato di particolare disordine amministrativo e di difficoltà finanziarie $^{\varsigma_{3}}$. L'infante Alfonso dovette imbattersi nei problemi di questo cenobio mentre era ancora impegnato nell'assedio di Iglesias: qui gli era giunta la supplica di frate Pietro de Brandis, nominato priore di San Saturno dalle autorità del monastero marsigliese ma il cui incarico non era, come si arguisce dal tenore del documento, pacificamente riconosciuto, che chiedeva per sé e per il monastero la protezione delle autorità aragonesi e la possibilità di godere integralmente dei redditi e dei diritti del priorato ${ }^{54}$. Il documento potrebbe sottintendere i difficili rapporti esistenti con i Pisani di Castel di Cagliari che non avevano mai visto di buon occhio la presenza del monastero e delle sue vaste proprietà nelle immediate vicinanze del Castello, e che in un momento particolarmente critico come quello precedente lo scoppio della guerra con i Catalani, avevano inteso tenere maggiormente sotto controllo i monaci attraverso due procuratori pisani incaricati di amministrarne $\mathrm{i}$ beni ${ }^{\text {ss. }}$. In quel difficile frangente, dunque, quando la guerra stava per spostarsi dal territorio iglesiente al Cagliaritano, il monastero di San Saturno si poneva sotto la protezione della Corona e, data la sua posizione strategica tra il Castello di Cagliari e il colle di Bonaria, venne inglobato nelle mura fatte costruire da Alfonso intorno al suo

\footnotetext{
${ }^{53}$ Per le vicende del priorato cagliaritano di San Satumo nel corso dei secoli $e$, in particolare, per le vicissitudini allraversate nel periodo pisano, A. BOSCOLO, L'abbazia di San Vittore cit.; F. ARTIZZU, Betlo Alliatc e alcuni possessi vittorini nel cagliaritano, in "Studi sui Vittorini in Surdegna" cit., pp. 9-12.

${ }^{54}$ ACA, Canc., reg. 396, ff. 57-58.

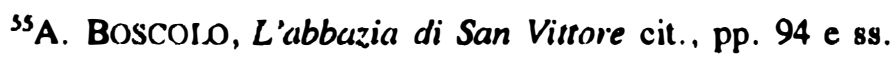


accampamento, diventando un caposaldo del sistema difensivo aragone$\mathrm{se}^{\mathrm{s6}}$.

Durante la guerra pisano-catalana il monastero subl danni gravissimi: se ne percepisce un'eco nella richiesta di giustizia fatta all'infante Alfonso dal nuovo priore Bertrando Isnard, il quale lamentava lo stato disastroso degli edifici, che rendeva impossibile persino la celebrazione delle sacre funzioni, e l'arbitraria occupazione di molte terre ${ }^{57}$. I problemi relativi alle proprietà terriere del monastero sono quelli che maggiorment: emergono dai documenti raccolti: con la conquista aragonese il patrimonio del priorato di San Saturno, che era ancora notevolmente esteso in tutto il Campidano di Cagliari ${ }^{58}$, continuo, infatti, ad assottigliarsi a vantaggio dei nuovi arrivati. I quali, approfittando della confusione creata dalla guerra e dall'espulsione dei Pisani dal Castello di Cagliari, miravano ad impadronirsi delle terre, situate nelle vicinanze della città e coltivate ad orti e vigne, che un tempo erano appartenute al monastero e che i monaci avevano venduto o concesso in enfiteusi, talvolta in modo assai poco vantaggioso, ad abitanti del Castello. Anche i religiosi, dal canto loro, tentavano di rientrare in possesso delle loro proprietà, cosicchè numerose erano le controversie e le richieste di giustizia rivolte al sovrano ${ }^{59}$. Molte terre del cenobio vittorino erano comunque entrate a far parte dei possessi feudali dei nobili iberici che avevano partecipato alla campagna di conquista, ai quali il sovrano stesso le aveva assegnate. La villa di Sinnai e il territorio denominato Campo di San Saturno, per esempio, cosl come altre terre con servi ed ancelle, da tempo immemorabile appartenenti ai monaci vittorini, erano state concesse a nobili catalani, tra i quali spiccava Berengario $\mathrm{Ca}$ rroz, che veniva accusato anche di aver contribuito alla distruzione del monastero portandosi via le pietre che poi aveva usato per la costruzione

\footnotetext{
55.

${ }^{36}$ G. MELONI, L'lialia medioevale nella cronaca di Pietro IV d'Aragona, Cagliari 1980, p.

${ }^{57}$ ACA, Canc., reg. 375, ff. 57v-58.

${ }^{30} \mathrm{Cio}$ si evince dal dettagliato inventario compilato nel 1338 dal priore Guglielmo de Bagamis, E. BARATIER, L'inventaire des biens du prieuré Saint-Satumin de Cagliari dependant de l'abbaye de Saint-Victor de Marseille, in "Studi storici in onore di Francesco Loddo Canepa", vol. II, Firenze 1959, pp. 41-74. Sull'utilizzazione e lo sfruttamento del patrimonio terriero viltorino C. MANCA, Aspetti dell'economia monastica vittorina nel Medioevo, in AA.VV., Studi sui Vittorini cit., pp. 55-88.

${ }^{50}$ ACA, Canc., reg. 316, f. 4 v.; reg. 403, ff. 196-197; reg. 508, ff. 96-96 v.; reg. 1007, ff. $154-154 \mathrm{v}$.
} 
del suo castello ${ }^{60}$. Delle difficoltà economiche dei Vittorini aproffittavano spesso mercanti e altri abitanti catalani di Cagliari i quali, nella frequente impossibilità di farsi restituire le somme di danaro, spesso cospicue, prestate ai monaci per le loro necessita, si impadronivano, anche con l'assenso regio, di terre del monastero. Era quanto pretendevano due cittadini iberici, Arnaldo Boscan e Bernardo de Ponte, che si erano rivolti al sovrano per avere riconosciuto il loro diritto ad essere risarciti, in un modo o nell'altro, dai monaci ${ }^{61}$. Alle rimostranze dei religiosi Alfonso rispondeva promettendo appoggio e protezione; in realtà la fame di terre dei nuovi abitanti di Cagliari e dei feudatari iberici era tale che la promessa non poteva risultare che priva di contenuto. E' emblematico dell'atteggiamento assunto dalla Corona riguardo ai problemi dei monaci l'ordine dato dall'infante Alfonso agli ufficiali regi incaricati di dirimere le controversie tra i Vittorini e i due cittadini iberici già citati: cio che premeva maggiormente al sovrano era che coloro che erano venuti in Sardegna per esercitarvi l'attivita mercantile o vi si erano trasferiti per popolarla non venissero disturbati da eccessivi problemi e difficolta burocratiche "... nec eciam pati velimus quod mercatores ac alii qui ad dictam insulam tam mercandi vel populandi se trasferunt, per anfractus litium ac alias iuris sollempnitates laboribus seu expensis aliisque indebitis vexacionibus fatigentur, sicque in premissis et similibus vos taliter habere curetis quod dictus supplicans ac alii subditi nostri per premissis atque similibus nullam iustam decetero habeant de vobis materiam conquerendi..." 62

Lo stesso problema della perdita delle terre si ritrova per gli altri monasteri situati nei territori conquistati dai catalano-aragonesi: per quanto riguarda il Cagliaritano i documenti finora esaminati danno notizia soltanto di un altro monastero, quello femminile di San Giorgio o di Santa Greca, presso Decimomannu ${ }^{63}$. Fin dal 1327 la badessa si era rivolta all'infante

\footnotetext{
${ }^{00}$ ACA, Canc., reg. 508, if. $85-85$ v. Su queste vicende cfr. L. D'ARIENZO, San Satumo di Cagliari e l'ordine militare di San Giorgio de Alfama, in "Arhivio Storico Sardo", XXXIV1 (1983), pp. 43-80. Sul ramo sardo della fauniglia Carroz L.L. BROOK, F.C. Casula, M.M. Costa, A.M. OLJVA, R. PAVONI, M. TANGHERONI, Genealogie medioevali di Sardegna, tav. XXXIV, Cagliari-Sassari 1984.

${ }^{61}$ ACA, Canc., reg. 403, f. 192v.; ff. 196v.-197.

${ }^{62}$ ACA, Canc., reg. 403, f. 192v.; ff. 196-197. Le difficoltà nel ripopolamento di Cagliari, che portavano l'infante Alfonso a chiedere un trattamento particolarmente favorevole per i Catalani che stabilivano il loro domicilio nell'isola, sono delineate da R. CONDE I Delgado de Moinna, Castel de Caller cit.
}

${ }^{63}$ Le uniche menzioni di questo monastero erano finora quelle, relative agli anni 1342 . 
Alfonso denunciando l'occupazione di beni e redditi da parte di heretats catalani, con grave danno per la comunità monastica. L'Infante, ricordando il preciso impegno di rispettare e difendere i beni della Chiesa, che i monarchi catalani si erano assunti al momento dell'infeudazione del Regno di Sardegna, "attendentes quod personas religiosas et eorum bona sub nostra dicione constituta tenemur in iure suo defendere et tueri", ordinava al governatore generale di rendere prontamente giustizia alle monache ${ }^{64}$. L'ordine, tuttavia, non venne rispettato se nel 1355, approffittando della presenza del re Pietro IV a Cagliari, la badessa di Santa Greca esponeva con maggiori dettagli lo stesso problema al sovrano, producendo anche la documentazione attestante che, per donazione perpetua del giudice Torchitorio e di altri sovrani cagliaritani, le ville di Arili e di San Venesio, occupate rispettivamente dal vicario del comune di Pisa e dal catalano Francesc de Sant Climent, appartenevano al monastero. La badessa denunciava anche gli abusi commessi dai feudatari nei confronti degli abitanti di quelle ville e dei servi del monastero ai quali gli stessi giudici di Cagliari avevano concesso determinati privilegi e che venivano invece costretti a indebite prestazioni lavorative ${ }^{65}$.

1347, contenute nelle Rationes Decimarum cit., pp. 56, 107, 156, 173. Non sappiamo a quale ordine appartenessero le monache che lo abitavano. Sulla chiesa di Santa Greca, annessa al monastero, R. CORONEO, Archilettura romanica dalla metd del Mille al primo '300, Nuoro 1993, pp. 38-39.

${ }^{6}$ ACA, Canc., reg. 403, ff. 199v-200. La perdita della giurisdizione su terre e servi a causa dell'introınissione di ufficiali regi o di feudatari era uno degli aspetti più significativi della profonda decadenza del inonachesiino tradizionale, come sottolinea anche M. RIU, El monaquismo catalan en el siglo XIV , in "Anuario de Estudios medievales", 7 (1971-1972), pp. 593-613. Sulle condizioni dei servi dei monasteri B. Fols, Territorio e paesaggio agrario nella Sardegna medieovale, Pisa 1990, pp. 132 e ss.

${ }^{65}$ ACA, Canc., reg. 1024, f. 101v. La villa di Arili (Arixi) si trovava nella curatoria di Trexenta, lasciata in feudo ai Pisani, insieme a quella di Gippi, dopo la pace del 1326, efr. F.C. Casula, Giudicati e curatorie cit., p. 102, mentre la villa di Sant Venes (Santu Inesu) era ubicata nella curatoria di Decimo, Ibideın, p. 99. Il documento é interessante anche per la citazione della documentazione giudicale esibita dalla badessa Giovanna per comprovare la proprieta delle terre e ripropone il problema degli archivi dei monasteri, che dovevano essere ricchi di documentazione tale da consentire una ricostruzione adeguata della consistenza patrimoniale e delle vicende storiche dei vari cenobi, e dei quali non ci sono rimasti che pochi documenti e alcuni registri patrimoniali risalenti al XIII secolo, i coseddetti "condaghi" (G. BONAZZI, Il corddaghe di San Pietro di Silki. Testo logudorese dei secc. XII-XIII, Sassari 1900; R.DI TUCCI, Il condaghe di San Michele di Salvenor, Cagliari 1912; Il condaghe di Santa Maria di Bonarcado, rist. del testo di E. BESTA riveduto da M. VIRDIS, Oristano 1982; P. MERCI, Il condaghe di San Nicola di Trullas, Sassari 1992) Ogni monastero doveva possedere più di un condaghe se, come risulta da un documento del 1280, nell'archivio del priorato di San Nicola di Trullas ne erano conservati ben cinque (G. ZANETTI, I Camaldolesi cit., doc. XVIII, pp. XLIV-XLVII). Non é inverosimile che, oltre all'incuria, alle devastazioni dovute 
Ancora più difficile era la situazione delle numerose fondazioni monastiche benedettine della Sardegna settentrionale. Con la conquista iberica il Logudoro divenne una delle zone più turbolente dell'isola: nonostante l'iniziale accordo con i catalano-aragonesi, dopo appena un anno Sassari e le famiglie Doria e Malaspina, proprietarie di vasti territori ${ }^{66}$, si ribellarono: mentre la città venne temporaneamente ridotta all'obbedienza con l'espulsione dei suoi abitanti e il ripopolamento con cittadini iberici67, i rapporti con le due famiglie attraverarono fasi alterne, più spesso conflittuali, sfociando non di rado in gravi scontri armatios. I monasteri dovettero subire le conseguenze di questa instabile situazione politica e militare: la riscossione dei tributi e dei fitti dovuti dagli affittuari divenne sempre più difficile, molte terre vennero abbandonate e lasciate incolte, mentre l'introduzione del sistema feudale e l'arrivo dei nuovi pobladors di Sassari ai quali, come incentivo al trasferimento in Sardegna, furono assegnate molte terre strappate ai patrimoni ecclesiastici, portarono a un'ulteriore riduzione dei loro possessi.

Le difficoltà economiche, la perdita di potere, di influenza e di prestigio emergono chiaramente dai documenti riguardanti $i$ monasteri logudoresi, alcuni dei quali erano stati tra i più ricchi e fiorenti dell'isola, come le abbazie di San Michele di Plaiano, dell'ordine toscano di Vallombrosa, situata nella curatoria di Romangia, non lontano da Sassari; di Santa Maria di Paulis, cistercense, nella curatoria di Coros, presso Ittiri; di SantaMaria di Tergu, cassinese, nella curatoria dell'Anglona, tra Osilo e Cas-

alle guerre e ai fattori ambientali, nella distruzione degli archivi dei monasteri abbia avuto una parte non irrilevante anche la volontà dei nuovi dominatori di eliminare le prove di proprictà delle terre di cui si erano impadroniti. Sulla dispersione di un altro archivio monastico, efr. P.F. SimBULA, L'archivio del monastero di San Martino di Oristano e la falsa donazione di Mariano IV d'Arborea, in "Medioevo. Saggi e rassegne" 18 (1993), pp.141-163.

${ }^{60}$ Su queste famiglie L.L. BrooK, F.C. Casula, M.M. Costa, A.M. OuVA, R. PAVoni, M. TANGHERONI, Genealogie cit., tav. XIX-XXV.

${ }^{67}$ Sulla rivolta antiaragonese scoppiata a Sassari nel 1329 e il ripopolamento della citta con cittadini iberici A.M. ARAGO CABAÑAS, La repoblacion de Sàsser bajo Alfonso el Benigno (1330-1336), in Atti del "VI Congreso de Historia de la Corona de Aragon", Madrid 1959, pp. 539-549; G. PALA, Una nota sul ripopolainento di Sassari al tempo di Alfonso il Benigno, in "Anuario de Estudios Medievales", 10, 1980, pp. 853-870; A. CASTEllaccio, Politica, economia e societd a Sassari nei primi anni della dominazione aragonese, in Aspetti di storia italo-catalana, Cagliari 1983, pp. 73-99.

${ }^{68}$ Su tutti questi avvenimenti F.C. CASULA, La Sardegna aragonese cit., vol.I, p. 213 e 88. 
telsardo; della Santissima Trinità di Saccargia, camaldolese, nella curatoria di Ploaghe, presso Codrongianos ${ }^{69}$.

Al momento particolarmente critico attraversato dall'abbazia di Plaiano subito dopo l'arrivo dei Catalano-Aragonesi nel Logudoro si deve attribuire, probabimente, l'iniziativa presa dal pontefice Giovanni XXII nel 1325 , di porre a capo dell'abbazia, dopo la morte dell'abate Ruggero, un amministratore apostolico di sua scelta nella persona, prima, dell'abate Corrado del monastero di San Benedetto di Muleggio presso Vercelli e, alla morte di questo, di frate Ubertino dello stesso monastero ${ }^{70}$. Anche Ubertino, che prese possesso del monastero nel 1330 , in un periodo particolarmente difficile per le conseguenze della rivolta sassarese, dovette incontrare non poche difficoltà nell'amministrazione dei beni dell'abbazia, soprattutto nel far fronte alle mire dei Catalano-Aragonesi sulle sue proprietà fondiarie. Una fetta del patrimonio terriero del monastero di San Michele di Plaiano finl ben presto nelle mani dello scrivano di Corte Miquel Amarell il quale dovette pero sostenere una lite con alcuni abitanti della villa di Geriti che si rifiutavano di versargli certi diritti; lo stesso personaggio si approprio anche di vasti territori appartenenti all'abbazia cistercense di Santa Maria di Paulis, approfittando delle difficoltà finanziarie dell'abate al quale aveva prestato una cospicua somma di danaro ${ }^{71}$. Nelle controversie che sorgevano in casi come questi il sovrano, chiamato in causa, pur ordinando agli ufficiali regi di esaminare eventuali documenti e di agire secondo giustizia, non poteva, come già si é visto, che favorire i suoi fedeli sudditi, a scapito dei monasteri.

Alla protezione del pontefice dovette ricorrere nel 1336 l'abate di Santa Maria di Paulis, stanco per le continue molestie, le violenze, le usurpazioni "castrorum, villarum, terrarum et aliorum locorum iurisditionum,

\footnotetext{
${ }^{69}$ Su queste abbazie G. ZANEרT1, I Vallombrosani cit.; I Cainaldolesi cit.; AA.VV., I Cistercensi cit.; A. SAJA, Montecassine cit. Per l'ubicazione delle curatorie F.C. Casula, Giudicati e curatorie cit.

${ }^{70}$ D. SCANO, Codice Diplomatico cit. vol. I, doc. CCCLXIX del 31 dicembre 1325; doc. CCCLXXIX del 26 febbraio 1328; J. TRENCHS-R. SÁNZ DE LA MAZA, Documentos Pontificios cit., doce. 5-8, 20, 32, 93, 94, 95. Sul più frequente intervento papale nell'amministrazione delle comunità monastiche alla line del Medioevo, G. PENCO, Storia del monachesimo cit., pp. 274 e $88 ., 373$ e ss.

${ }^{71}$ ACA, Canc., reg. 515, f. 79. La villa di Geriti o Geridu si trovava nella curatoria di Romangia, cfr. F.C. CASUlA, Giudicati e curatorie cit., p. 108, e faceva parte del territorio della città di Sassari, P.TOLA, Codex cit., tomo I, parte II, doc. XXVII.
} 
iurium et bonorum mobilium et immobilium"72 che venivano perpetrate non soltanto dai feudatari iberici e dagli ufficiali regi ma anche dai Doria e dai Malaspina; al vicario di quest'ultima famiglia, padrona di territori nella curatoria di Coros, venne attribuito, infatti, il brutale assassinio dello stesso abate di Paulis causato, verosimilmente, da controversie di carattere territoriale $^{73}$.

Le conseguenze della rivolta di Sassari e del ripopolamento della città con cittadini iberici colpirono duramente i monasteri della zona: in mano a Catalani erano andati, per esempio, numerosi mulini che si trovavano nella valle di Bosoe, a nord-ovest di Sassari. Mentre fino al tempo dell'espulsione dei Sassaresi i proprietari dei mulini erano soliti pagare al monastero di Plaiano un censo annuale per l'utilizzazione dell'acqua del fiume sul quale sorgevano, censo che era dovuto ai monaci per antica concessione dei giudici turritani, i nuovi proprietari si rifiutavano di pagare quel tributo, con grave danno economico per il monastero ${ }^{74}$. Ugualmente erano state occupate da Catalani vigne terre ed altre proprietà dell'abbazia cassinese di Santa Maria di Tergu situate nelle curatorie di Romangia, Flumenargia e nella baronia di Osilo ${ }^{75}$.

Ai disordini succeduti alla rivolta del 1329 fu dovuto, presumibilmente, anche l'intervento di Alfonso il Benigno nei confronti del monastero femminile di San Pietro di Silki, situato alle porte della città turritana, che aveva visto molte sue terre occupate da ribelli sassaresi. L'importanza di quei territori e la precarietà della situazione indussero il sovrano ad affidare l'amministrazione del monastero ad una persona di sua fiducia, il suo capellano Pere de Deu, monaco cistercense catalano, neo-eletto vescovo di Santa Giusta ${ }^{76}$.

Una situazione di grave difficoltà economica veniva lamentata nel 1333 anche dal priore del monastero vittorino di San Nicola di Guzule,

\footnotetext{
${ }^{72}$ D. SCANO, Codice Diplomatico cit., I, doc. CDXXXVIII.

${ }^{73}$ ACA, Canc., C.R.D. di Alfonso III il Benigno, cassa 21, doc. 3261; reg. 1007, ff. 235v.-236; reg. 1016, f. 53v.; reg. 1024, ff. 70-70v., 100-100v.

${ }^{74} \mathrm{ACA}$, Canc., reg. 1016, ff. 53v-54. Sull'utilizzazione del mulino ad acqua nella Sardegna medioevale B. Fols, Territorio e paesaggio agrario cit., pp. 121 e ss.

${ }^{75}$ ACA, Canc., reg. 1020, f. 51.

${ }^{76} \mathrm{ACA}$, Canc., reg. 511 , f. 3 v. Sulla nomina di questo personaggio a vescovo di Santa Giusta, diocesi del giudicato d'Arborea, ACA, Canc., reg. 510, ff. 179v.-180; reg. 511, ff. 33v.; reg. 512, ff. 235-235v.; J. TRENCHS-R. SÁINZ DE LA MAZ.A, Documentos pontificios cit., p. 20 e docc. elencati nell'indice a p. 207.
} 
presso Ozieri ${ }^{n}$ : fin dal periodo precedente l'espulsione dei sassaresi, alcuni abitanti di questa città tenevano arbitrariamente occupati terre e salti del monastero situati nel territorio di Querqui ${ }^{78}$. La perdita di questi possessi, che comprendevano anche la chiesa di Santa Maria, appartenente al monastero, aveva portato il priorato di San Nicola "ad maximam penuriam ac eciam paupertatem"79.

Nonostante le rimostranze dei religiosi e le promesse regie di protezione e di giustizia, le condizioni dei monasteri benedettini continuarono a deteriorarsi. Nel 1355, approfittando della presenza nell'isola del re Pietro IV il Cerimonioso giunto a Cagliari per convocare le prime Corti generali del Regno ${ }^{80}$, numerosi abati si recarono personalmente presso il sovrano per denunciare una situazione di disagio che perdurava ormai da decen$\mathrm{ni}^{81}$.

La conquista aragonese fu comunque fatale agli antichi cenobi benedettini sardi, già da anni investiti da una profonda crisi, e contribul ad accelerarne la scomparsa che avvenne, nella maggior parte dei casi, prima della metà del XV secolo. Nei confronti della loro sorte, segnata dagli eventi e dal mutare dei tempi, non ci fu, da parte del potere politico aragonese, che un sostanziale disinteresse ${ }^{82}$.Il nuovo assetto politico e territoria-

\footnotetext{
${ }^{77}$ Per le vicende di questo priorato A. Boscolo, Il priorato vittorino di San Nicola di Guzule, in "Studi sui Vittorini in Sardegna", Padova 1963, pp. 25-35.

${ }^{78}$ La villa di Querqui (Kerki o Chcrcos) si trovava nella curatoria di Fluminargia e faceva parte delle pertinenze della città di Sassari. L'infunte Alfonso l'aveva concessa in feudo al sassarese Margherito Rapallino ma, dictro richiesta dci sassaresi il 19 aprile 1324 aveva revocato la concessione perché contraria ai privilegi della citta, P. TOLA, Codex cit., tomo I, parte II, doc. XXVII. Lo stesso Margherito Rapallino, insieme a Torgodorio Puligue, é accusato dai monaci vittorini di aver occupato arbitrariamente le terre del monastero di San Nicola di Guzule situate nel territorio di quella villa.

${ }^{79}$ ACA, Canc., reg. 516, ff. 163-163v.

${ }^{80} \mathrm{Su}$ questo importante avvenimento Il Parlamento di Pietro IV d'Aragona (1355), a cura di G. MELONI, II volume degli Acta Curiaruin Regni Sardiniae, Cagliari 1993.

${ }^{81}$ ACA, reg. 1018, f. 94 v.; reg. 1024, f. 100; reg. 1025, f. 124.

${ }^{82} \mathrm{Su}$ un'eventuale catalanizzazione dei vertici dei principali monasteri benedettini abbiamo dati ancora più scarsi che per gli Ordini Mendicanti. Tultavia qualche tentativo in questo senso venne probabilmente attuato: catalano doveva essere, per esempio frate Giovanni, abate di Santa Maria di Paulis, che il re raccomandava al govermatore dell'isola nel momento in cui prendeva possesso del monastero (ACA. Canc., reg. 1018, f. 94v.) o frate Guglielmo Vassal, abate di Santa Maria di Tergu, che ebbe vari incarichi durante l'assedio di Alghero del 1354 e per il quale venne chiesto un vescovado che lo ricompensasse per i suoi meriti, ACA, Canc., reg. 1024, ff. 12-12v.; f. 16; f. 22; L.D'ARIENZO, Carte Reali cit., doc. 548, pp. $281-282$.
} 
le imposto all'isola dopo la conquista mise $i$ sovrani iberici nell'impossibilita di tenere fede all'impegno di rispettare e difendere i beni della Chiesa preso da Giacomo II per sé e per i suoi successori al momento dell'infeudazione del Regno di Sardegna e Corsica, e le proprietà immobiliari dei monasteri, cosl come quelle degli altri enti ecclesiastici possidenti, vennero smembrate, depredate e sottoposte alle giurisdizioni feudali.

La politica catalano-aragonese nei confronti degli ordini religiosi nella prima meta del XIV secolo, pur guidata da precisi interessi, non poteva, in definitiva, che rispecchiare la realta religiosa e sociale del tempo, che vedeva negli ordini monastici benedettini il retaggio di un mondo in declino e negli Ordini Mendicanti l'espressione delle nuove forze sociali ed economiche e delle nuova sensibilità religiosa.

\section{RÉSUMÉ}

Le roi catalan Jacques II et son fils, l'Infant Alphonse, en entreprenant la conquête de la Sardaigne pisane, croyèrent de fondamentale importance obtenir l'appui du clergé local. La documentation retrouvée dans les Archives de la Couronne d'Aragon met en évidence le considérable rôle attribué par les souverains catalano-aragonais aux Ordres Mendiants, surtout Franciscains et Dominicains, comme véhicule pour la catalanization de l'île, et les efforts faits pour s'en assurer la fidélité. La documentation démontre, en outre, comme l'occupation catalano-aragonaise marque le déclin définitif des Ordres Monastiques Bénédictins, dépuis longtemps en grave décadence, même si encore propriétaires de vastes extensions territoriales.

\section{SUMMARY}

The Catalan king James the Second and his son, the Infant Alfonso, thought that the support of the local clergy support was of fundamental importance in undertaking the conquest of the Pisan territories in Sardinia. The documents found in the Crown of Aragon Archives point out the im- 
portance attached by the Catalan-Aragonese sovereigns to the Mendicant Orders, especially the Franciscans and the Dominicans, as a vehicle for the Catalanization of the island, and the efforts made to gain their loyalty. The documents also show that the Catalan-Aragonese occupation marked the definitive decline of the Monastic Benedictine Orders, which had already largely decayed, although they were still possessors of vast territories. 